\title{
Las revistas latinoamericanas de Bibliotecología y Ciencias de la Información bajo el prisma de los servicios bibliográficos internacionales
}

\author{
Héctor Gómez Fuentes*
}

\section{RESUMEN}

Elartículopresentaunavisióngeneraldelosproblemasdelaliteraturabibliotecológicachilenay un es tu dio de los me dios de di fu sión de las re vis tas la ti no ame rica nas en Bi blidecologíay Cien cias de la In for ma ción, so bre la base del re gis tro de sus ar tículosen elLibrary\& Information Science Abstracts, Information Science Abstracts y Library Literature.

\section{ABSTRACT}

Thisarticle presentsagene ral vision of the problems oflibraryscien celite rature in chi le and a study of the means of diffu sion of the latin American magazines in the "Bibliote cológica y Ciencias de la In for ma ción" (library and In for ma tion Scien ces). This is ba sed upon the re gister of its articles in Library \& Information Science Abstracts, Information Science Abstracts and Library Literature.

\section{INTRODUCCION}

En el mes de octubre de 1992 se realizó Clareunión de trabajo "PresenteyFutur ro de INFOBILA" en el Cen troUniversitario de Investigaciones Bibliotecológicas (CUIB), en la Ciudad de México, D.F., con el patrocinio de IFLA/Advancement of Librarianship in The Third World Programme. En el marco deestare unión se abor dó elproblemadelaliteraturabibliotecológica latinoamericana. Entre sus objetivos estaban los de pro cu rar res pon der a lassiguientes interrogantes: ¿Quién produceliteratur ra bibliotecológica? ¿Quiénes la usan y para qué la usan? ¿Cómo se distribuye y adquiere?.1
En el caso de Chile, me atrevería a afirmar que los "productores de literatura" son aquellasinstitucionesoindividuosquese dedican a pensar nuestra profesión, a realizar estudios, a presentarproyectos o a resolver problemas prácticos que plantean, por ejemplo, la introducción de nuevastecnologías, ladifusióny promociónde los serviciosbibliotecarios, lacapacitación, etc.

Como dato cu rio so, y lue go de un aná lisis de los 297 documentos registrados en la bibliografía Literatura BibliotecológicaChilena1980-1992,2 se des prende que cuatro son las profesiones más produc ti vas en lo que a li te ra tu ra de in- terésbibliotecológicose refiere:Bibliotecarios $(54,88 \%)$, Ingenieros (13,53\%), Médi $\cos (6,01 \%)$, Profesores $(4,51 \%)$.

Las últimas tres categorías de profesionalesautores, participan en actividades de interés biblio te cológico en lasáreas de la tecnología delainformación, comunicacióncientífica, epistemometría, servicios de información especializadas, hábitos de lectura, etc.

Paradójicamente, en el caso chileno y por lafal tadeun me dio de comunica ciónespe cializado estable, la literaturade interés bibliotecológicoseencuen tra dispersay, sue le encontrarse en una amplia variedad de 
revistas médicas, deeduca ción, de historia y de ingeniería, entre otras.

En Chile, al no exis tir revis tas de la especialidad, el mo de lo de dispersión de Bradford no se cumple, y no existe una concentración en publi ca cio nes nu cleares. Sólolarevistase mestral "Trilogía", órgano oficialdel Instituto Profesional de Santiago, el cual alberga a la Escuela de Bibliotecología, suele publicar trabajos con relativa frecuencia.

En el área de la Bibliotecología, en Chile, son escasas las publicaciones formales, ya sea en for ma de mo no gra fías o de re vis tas, queseanelproductodeuntrabajoeditorial rigurosoqueasegurelacalidaddelconteni do. Sin embargo, se produceunacantidad de documentos de valor para la profesión, que noson publicadosformal men tey cuya distribución es limitada.

Como dato curioso, algunos bibliotecarios chilenos se han preocupado de elaborar nor mas de presen ta ciónyedi ción de publicaciones periódicas y seriadas, dando origen a guías y ma nua les de edi ción, que utilizan otros profesionales en su actividad editorial. ${ }^{3}$ No obstante, hemos sido incapaces, de publicar una re vis ta chile nade la especialidad. Sólo es posible contar con nuevepublicaciones de difusiónenelárea, caracterizadas por su pobre calidad de impresión, irregularidad y su limitado tiraje.

Como una ex cep ción que sólo con fir ma la regla, recientementefuepublicadouninte resante libro sobre "Análisis y Recupera ción de Información Documental", cuya au tora eslaSra. Texia Igle sias, profesorati tular de la Escuela de Bibliotecología. ${ }^{4}$

Todo lo anterior, nos lleva a afirmar que iMás que productores, somos consumidores de literatura formal!; y un espacio no despreciable dentro de medio académico enquemedesenvuelvo, loocupalalectura derevistaslatinoamericanas de bibliotecología y ciencias de la información. Una visión so bre es tasúl ti mas es lo que tra ta réen este artículo.

\section{LAS REVISTAS LATINOAMERICANAS DE BIBLIOTECOLOGIA Y CIENCIAS DE LA INFORMACION}

Seestimaenaproximadamente 1,000títur loslacantidaddepublicacionesperiódicas y seriadas en el área de Bibliotecología y Ciencias de la Información; y la cantidad de artículos publicados anualmente, excede los 15,000. 5 Sólo el Library and Information Science Abstracts, en su versión en CD-ROM (1969-Marzo 1992) incluye 112,626 registros que, en su mayor parte, provienen de un número aproximado de 550 revistas publicadas en más de treinta idiomas.
Ahora bien, una consultarápidaaThe SerialsDirectorybajo el tér mi no "Li brary and InformationSciences", nos entrega un númeroaproximadode 2,196acier tos, de los cuales 49 (2,45\%), son títulos publicados en países de América Latina. De estos últimos, sólo16 correspondenapublicaciones primarias de la especialidad, el resto son fuentes secundarias, directorios, boletines de bibliotecas, etc.

Esprecisamente a travésdelosdirectorios, como el nombrado anteriormente, como lo gra mos dar una respues ta for mal ala pregunta: ¿Qué revistas de Bibliotecología y Ciencias de la Información se publican en los diferentes países de América Latina?

\section{MEDIOS DE DIFUSION DE LAS RE- VISTAS LATINOAMERICANAS}

Los editores de revistas de la especialidad suelen utilizar medios ocasionales de difusión de nue vos tí tu los, como car tas a los lectores potenciales, catálogos, folletos, etc., anunciando la publicación de sus revistas.

Sin embargo, el conocimiento de la existencia de revistas latinoamericanas en BibliotecologíayCien ciasdelaInformación, selogra por la revisiónperiódica de directorioscomo elUlrich'sInternational PeriodicalDirectory, el Serials Directory, cuyas versiones en CD-ROM posibilitan la búsqueda por editorial, país, etc. Estas fuentes son instrumentos fundamentalesenlosdepartamentos de adquisición de una biblioteca universitaria o especializada.

La existencia de las re vis tas en los re per to rios mencionados, revela la preocupación de los editores por darlas a conocer a nivel mundial, actualizando periódicamente los datos de sus publicaciones, en los for mu la rios que en vían las em pre sas edi toras de estos directorios.

Es así como una búsqueda en estas fuentes, por país y bajo el tér mi no Li brary and In formationSciences, nos revelaloincompletas quesonenlacoberturaderevistaslatinoame ricanas. Por lo tanto, es necesaria la consulta complementaria, debido a que títulos que aparecen en el Ulrich's Plus no figuran en el SerialDirectory, yviceversa. (vertablaNo. 1). Además, se observa que aproximadamente un $70 \%$ delostítulos listados, correspondena revistas descontinuadas, y probablemente, por falta de iniciativa de los editores, no figuran publicaciones vigentes, como la Revista de Bibliotecología y Ciencias de la Información de Costa Rica e INFOLAC.

Un lugar aparte lo constituye, comofuente de se lec ción, el "ISSN Compact" es de cirla versión en CD-ROM del ISDS Register. En esta base de datos internacional de publicaciones periódicas y seriadas, es posible lo calizar las re vis tas con ISSNy títu lo clave, quehansidopublicadasenAméricaLatina en el área de la Bibliotecología y las Ciencias de la Información.

\section{MEDIOS DE DIFUSION DEL CON- TENIDO DE LAS REVISTAS L A T I - N O A M E R I C A N A S DE BIBLIO- TECOLOGIA Y CIENCIAS DE LA INFORMACION}

En el con tex to de la for ma ción del bi bliote cario-documentalista en la Escuela de Bibliote cologíadeSantiago de Chile, laenseñanza en el uso de las fuentes de informaciónenelárea delaBibliote cología y las Ciencias de la Información ocupa un papel importante.

Enlaasignaturade "Méto dos de InvestigaciónBibliográfica", en el 1er. año de la carrera, se de sarrolla una unidad sobreliteratura de la especialidad, que es de utilidad para los estudios e investigaciones que se llevan a cabo posteriormente en los seminariosy trabajosdeformulacióndeproyectos.

Es así como una búsquedadeinformación en el área, necesariamente pasa por una consul ta en el Li braryandInformation Abs tract, en el Library Literature y si se tiene acceso a la ver sión en CD-ROM el Information Science Abstracts.

Desgraciadamente, dos servicios de habla hispanaquecubrenlaliteraturabibliotecológica, INREB e INFOBILA, no han te ni do el impactoesperadoenelmediona cional. El primero, con una estructura de servicio de aler ta, re fle ja en su in te rior las ta blas de contenidodelasrevistaslatinoamericanas, yerararamenteconsultadoenlaBiblioteca denuestraEscuela. El segundo, disponible en $\mathrm{CD}-\mathrm{ROM}$ se en cuen tra enCONACYT y hasta ahora constituye un misterio para los especialistas chilenos.

\section{COBERTURA DE LA LITERATURA LATINOAMERICANA EN BASES DE DATOS BIBLIOGRAFICOS}

Ahorabien,surgelapreguntacExistelaposibi lidad de localizar y recuperar información publicada en revistas latinoamericanas, consultando las bases de datos de cobertura internacional? Una búsqueda en las bases de datos en CD-ROM: LISA PLUS, Information ScienceAbstractsyLibrary Literature, realizada con la estrategia de búsqueda: FIND: Título de la revista IN SO, nos presenta el resultado que se muestra en la Tabla No. 2.

De la ob serva ción de laTa blaNo. 2 se desprende que el Library and Information ScienceAbstracts, es la fuente de información que in diza el mayor nú me ro de artículos publicados en revistas latinoamericanas. Esto último, coincide con los resultados del estudio realizado en el año 1975, por la FID, y que fue posteriormente analizado en profundidad por el profesor Adolfo Rodríguez. 6 
Medios de difusión de las revistas latinoamericanas de Bibliotecología y Ciencias de la Información

\begin{tabular}{|c|c|c|c|}
\hline Título & País & $\begin{array}{l}\text { Serial Direc- } \\
\text { tory } 1992 \\
\end{array}$ & $\begin{array}{r}\text { Ul rich Plus } \\
1990-1991 \\
\end{array}$ \\
\hline 1. ALEBCI. Boletín Informativo & $\mathrm{MX}$ & - & ++ \\
\hline 2. AMBAC Noticiero & $\mathrm{MX}$ & ++ & ++ \\
\hline 3. AIBDA. Boletín Informativo. & $\mathrm{CR}$ & - & ++ \\
\hline 4. Anuario de Bibliotec., Archivología e Informática & $\mathrm{MX}$ & ++ & - \\
\hline 5. Archivo de ABIESI & $\mathrm{MX}$ & ++ & - \\
\hline 6. Associaçao Bahiana de Bibliotecarios, Informa. & $\mathrm{BR}$ & - & ++ \\
\hline $\begin{array}{l}\text { 7. Asociación Ex-Alumnos. Escuela Nacional de Bi- } \\
\text { bliotecarios. }\end{array}$ & AR & - & ++ \\
\hline 8. Bibliotecas & $\mathrm{CU}$ & - & ++ \\
\hline 9. Bibliotecología y Documentación & AR & ++ & - \\
\hline 10. Boletín ABDF & $\mathrm{BR}$ & ++ & - \\
\hline $11-C B$ & $\mathrm{MX}$ & ++ & - \\
\hline 12. Ciencia da Informaçao & $\mathrm{BR}$ & ++ & ++ \\
\hline 13. Cinterfor Documentación & UY & - & ++ \\
\hline 14. Documentación Bibliotecológica & AR & ++ & ++ \\
\hline 15. Documentaçao e Informaçao & $\mathrm{BR}$ & - & - \\
\hline 16. Estudos Avancados en Biblio. & $\mathrm{BR}$ & ++ & - \\
\hline 17. Papiro. Rep. Dominicana & $\mathrm{DO}$ & - & ++ \\
\hline 18. R. da Escola de Biblio UFMG & $\mathrm{BR}$ & ++ & ++ \\
\hline 19. R. Biblioteconomia de Brasilia & BR & ++ & ++ \\
\hline $\begin{array}{l}\text { 20. R. Brasileira de Biblioteconomia e Documen- } \\
\text { taçao }\end{array}$ & $B R$ & - & ++ \\
\hline 21. R. Latinoamericana de Documentación & BR & ++ & - \\
\hline 22. R. de ASCOLBI & $\mathrm{CO}$ & ++ & - \\
\hline 23. R. Interamericana Bibliotecología & $\mathrm{CO}$ & ++ & ++ \\
\hline 24. R. AIBDA & $\mathrm{CR}$ & ++ & ++ \\
\hline $\begin{array}{l}\text { 25. R. Escuela Universitaria de Bibliotecología. F. } \\
\text { Capurro. }\end{array}$ & UY & ++ & - \\
\hline 26. R. deL SINASBI & $\mathrm{VE}$ & ++ & - \\
\hline
\end{tabular}

A los factores anteriores, se agrega que estos servicios de indización incluyen las revistasque hemosseñalado, después deque sehanpublicadovariosvolúmenes; suelen cometer errores de transcripción en los datos identificatorios de los artículos indizados (Ver LISA 89/3652) y, muy frecuentemente, norespetanlasnormas deen tradas de au toresdeorigenespañol (iMoraleja, si es au tor, y quie re apa re cer en elAuthor Index como corresponde, separe sus dos apellidos por un guión o identifíquese con sólo el apellido paterno!).

Otro aspecto a considerar, es el factor tiempo. Vale decir, cuánto hay que esperar para que los artículos publicados en revistas latinoamericanas sean registrados en las basesdedatosmen cionadas. Estefenómenose conoce como operatividad de reflejo de la literatura, y se puede definir como el tiem po que pasa des de el mo men to de lapublicación del artículo, hasta su inclusión en una revista de índices y resúmenes.

Porejem plo, unservicio de alerta comoel $\mathrm{Cu}$ rrent Contents posiblementetieneunaoperati vi dad alta (uno a dos me ses aprox.); en cambio, un servicio exhaustivo y con resúmenes como el ChemicalAbstracts ten dría una operatividad más baja (cinco a nueve meses). La operatividad se calcula por la fórmula:

$$
\begin{gathered}
\mathrm{T}=\frac{-1 \mathrm{~N}_{1}+2 \mathrm{~N}_{2}+3 \mathrm{~N}_{3} \ldots \mathrm{MNm}}{\mathrm{N} 1 \mid+\mathrm{N} 2++\mathrm{N} 3 \ldots \mathrm{Nx}, \text { donde: }} \\
\mathrm{T}=\text { Operatividad promedio }
\end{gathered}
$$

$1,2,3, \ldots \mathrm{MNm}=$ intervalo de tiempo en meses, desde la publicación
Al respecto, el profesor Rodríguez señala que la pro duc ción del Ter cer Mun do nollegaapublica cionessecundariasporfal ta de una adecuada promoción y difusión y, cuando llegan, no las consideran con problemas idiomáticos y por un prejuicio con respecto a la calidad e importancia de su contenido.

Las revistas latinoamericanas que logran serin cluidasenserviciosbibliográficos, son parcialmentecubiertas; por ejem plo, de 16 artículos publicados en 1987 en la Revista da Escola de Biblioteconomía da UFMG, sólo 14 fue ron in di za dos en elLISA y de 12 publicadosen laRevistaInteramericanade Bibliotecología sólo 6 fueron incluidos en esta fuente secundaria. 7

Por otra par te, elLISA in dizalos 11 artículos publicados en Investigación Bibliotecológica en el año 1987, mien tras que los 16artículospublicadosenlarevistabrasileña, anteriormente nombrada, son in -

\begin{tabular}{|c|c|c|c|}
\hline \multicolumn{4}{|c|}{$\begin{array}{l}\text { Artículos de revistas latinoamericanas indizados en bases de datos bi- } \\
\text { bliográficos en Bibliotecología y Ciencias de la Información. } \\
1992 .\end{array}$} \\
\hline \multirow{3}{*}{ TITULO DE LA REVISTA } & \multicolumn{3}{|c|}{ BASEDEDATOS } \\
\hline & \begin{tabular}{|c|} 
ISA $(1969-\mathrm{mar}-$ \\
zo 92$)$
\end{tabular} & $\begin{array}{c}\text { ISA (1974- } \\
1992)\end{array}$ & $\begin{array}{l}\text { W ILSONLINE } \\
\left(\begin{array}{c}\text { (Dic. } 84-\text { Marzo, } \\
92)\end{array}\right. \\
\end{array}$ \\
\hline & No. art. & No. art. & No. art. \\
\hline 1. R. Biblioteconomia de Brasilia & 284 & 5 & 145 \\
\hline 2. Ciencia da Informaçao & 207 & 129 & 32 \\
\hline 3. R. Escola Biblioteconomia UFMG & 167 & 40 & 92 \\
\hline 4. R. Interamericana de Bibliotecología & 116 & 1 & 16 \\
\hline 5. R. AIBDA & 109 & 2 & - \\
\hline 6. R. Latinoamericana de Documentación & 29 & - & - \\
\hline 7. Investigación Bibliotecológica & 23 & - & - \\
\hline 8. R. Biblioteca Nac. José Martí & 6 & - & - \\
\hline 9. Documentación Bibliotecológica & 5 & - & - \\
\hline 10. R. Chilena Bibliotecología y Doc. & 4 & - & - \\
\hline 11. Boletín ABDF & 4 & - & - \\
\hline Total & 954 & 117 & 285 \\
\hline
\end{tabular}
cluidos en el Library Literature. 
delar tículo has ta su in clu sión en una re vis ta de resúmenes.

$\mathrm{N} 1, \mathrm{~N} 2, \mathrm{~N} 3 \ldots \mathrm{Nx}=$ nú me ro de ar tí culos publicados e incluidos en la revista de resúmenes.

To man do comobase eluniversodeartículos pu bli ca dos en el año 1987 por las re vistas men cio na das, más uno de la Revistade laBibliotecaNacionalJoséMartí y que fueron indizados en las fuentes secundarias mencionadas, se distribuyó cada artículo, según el intervalo de tiempo que demora en ser incluido en cada uno de los fascículos del LISA y el LL del año 1989. Como resultado se concluye que la operatividad promedio en el LISA y del LL es de 24 y 20 meses, respectivamente.

En otraspalabras, es ne ce sa rio esperarpacientemente los tiempos indicados para tener noti cias, a tra vés de al gu no de losservi cios bibliográficos mencionados, de la existencia de algún artículo de interés publicado en revistas latinoamericanas.

Porotraparte, estosserviciosbibliográficos internacionales ofrecen la ventaja de permitir localizar artículos de colegas latinoamericanos que publican en revistas extranjeras, muchas de ellas de prestigio in ter nacio nal. Es de cir, responden alapregun ta ¿quiénes en el país han publicadoen el extranjero?

Como dato anec dótico, lue go de con sultar el LISA, des pués de tres años me en te ré que una bibliotecaria chilena había publicado unartículosobrelaBibliotecaNacional, en la des co no cida para míre vistaAlexandria, editada, segúnlaautora, en GranBretaña.

Alrespectosugierolalecturadelartículode Budd, quien da indicaciones de cómo lograr que se le pu blique en re vis tas de pres tigio internacional. ${ }^{8}$

Probablemente, no estamos muy lejos de asumircomopatróninterna cionaldemedi ción de la productividad científica en el área de la Bibliotecología y Ciencias de la Información, el número de artículos publicadosen revistas de circula ción in ter nacional. Esta tendencia tan acentuada en el contexto de la comunidad científica, especial men te en el área de las cien cias exac tas y naturales,pareciera ser lapidariaparalas revistas científicas latinoamericanas.

A lo anterior se agrega el hecho de que se han erigi do como un "Be ce rro de oro", los in formes delJornalCitationReports del ISI.
Si aparece en el Cience Citation Index o Current Contents, es señal de que la publica ción in gresa enla "ór bita" de losestu dios de Garfield. 9 Paradójicamente, no es importantequeunartículoesté in dizadoenel Biological Abstracts, Chemical Abstracts o Engineering Idex, legendarias y prestigiosas fuentes de información mundial.

Invito a un estudio, para determinar quién citaaquiénenlabibliotecologíalatinoameri canaybuscarel "Clásico" de nuestraliteratur ra.Para esosugiero comenzarel es tu dio con el análisis del Social Science Citation Index.

Finalmente, y a modo de conclusión, y teniendo presente que sólo un buen sistema depublicacionesprimarias, aseguralacalidad de una fuente secundaria como es INFOBILA, se puede señalar lo siguiente:

1.En general, las revistas latinoamericanas sobrevivientes cumplen con las normas internacionales de presentación de revistas científicas. Sin embargo, existen elementosdeficitariosencuantoairregularidad en la publicación, pobreza en la calidad de la impresión, la tendencia a cumplir el rol de fuenteprimariaysecundaria a la vez, que les restaserie dadyles impide cumplir bien cualquiera de los dos roles.

2.Laaplicación denormas depresentación de revistas científicas (ISO/R8-1954, ISO/R 21-1961, ISO/214-1976), en algunasdelasrevistaslatinoamericanas, facilita la difusión de sus contenidosatravés de las bases de datos bibliográficos.

3.Si aplicamos cualquiera de los métodos de evaluación de revistas propuestas en la literatura diríamos que las siete primeras revistas que aparecen incluidas en las bases de datos analizadas, recibirían unacalificaciónentrebuenaasobresaliente. 10

4.Noobstanteloanterior, laspublicaciones latinoamericanas que logran ser incluidas en fuentes secundarias como el $\mathrm{Li}$ brary Literature y Library and Information Science Abstracts, lo hacen con una operatividad promedio de dos años.

5.Sólo un cuerpo responsable de editores, que tenga por misión evitar las que, según Arboleda son las características negativas de la literatura latinoamericana:

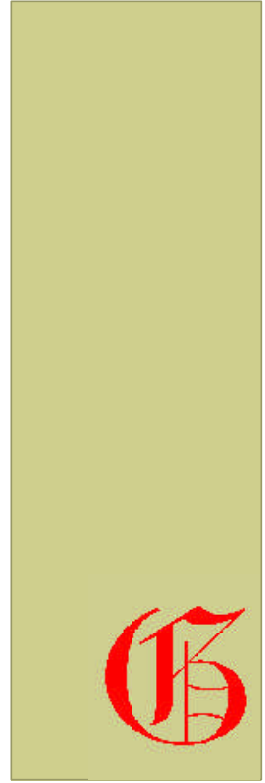

"En general, las revistas sobrevivientes cumplen con las normas internacionales de presentación de revistas científicas"

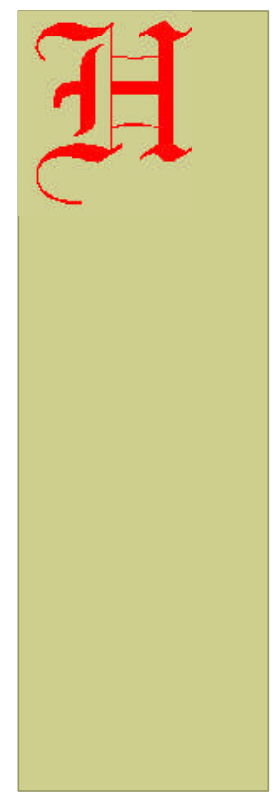


plagio, reproducción innecesaria, trabajos sin bibliografía consultada, terminología confusa, repeticióndetemasintrascendentes, ase gu ra rála cali dad de laspublicaciones que serán incluidas en INFOBILA. ${ }^{11}$

Finalmente, me sumo a la observación de uno de los participantes a la Mesa Redon- da, "Literatura Bibliotecológica y su uso", quien afirmaba que existeunmenosprecio por la literatura nacional, hay un prejuicio incorporado, que implicaunafal tadereconocimiento por el quehacer de los colegas latinoamericanos.
Sin embargo, tuve la grata experiencia de observarelinterésmanifestadopormiscolegas chilenos, cuando se exhibieron las publicaciones del CUIB en la XXII Feria InternacionaldelLibrorealizadaenSantiago de Chile, o cuando consulté un artículo de la revistaInvestigación Bibliotecológica.

\section{NOTAS}

1. REUNION DE TRABAJO: "Presente y futuro de INFOBILA". México, D.F., 5 al 9 de Octubre, 1992. Informe México, D.F., CUIB-UNAM/IFLA, $1992.7 \mathrm{p}$.

2. ESCUELA DE BIBLIOTECOLOGIA. Literatura Bibliotecológica Chilena 1980-1992. Santiago, I.P.S., 1992. 132p.

3. Alrespecto, colsú te se las obras de Gó mez, Héctor. Guía para la edi ción depublicacionesperiódicas.Santiago,EdicionesUniversidadCatóli ca de Chi le, 1985. 41 p.; y de Herbstaedt, El frie de. Publicaciones seriadascientíficas: un manualparasuedición. Santiago, Chile, CONICYT, 1988. 56p.

4. IGLESIAS, Texia. Análisis y recuperación de información documental. Santiago, Ediciones I.P.S., Chile. 1992. 237 p.

5. MARCO, Guy "Bibliographic control of Library and Information Science Literature". Libri, 33(1): 45-60, March, 1983.

6. RODRIGUEZ,Adolfo. "Laspublicacionesperiódicasdebibliotecología en los países en desarrollo". Investigación Bibliotecológica: archivonomía, bibliotecología e información, 1(2):3-14, Enero-Julio, 1987.

7. HUERTA, Luis, JuliaLeal. "LasrevistaslatinoamericanasdeBibliotecología y Cien cias de la In for ma ción y su re fle jo en ba ses de da tos bi bliográficos". Seminario de título. Profesor Guía: Héctor Gómez. Santiago, Escuela de Bibliotecología, IPS, 1990. 55 p.

8. BUDD, John. Publicar en bibliotecología y ciencia de la información. Revista Interamericana de Bibliotecología, 12(2): 71-91, Julio-Diciembre, 1989.

9. GARFIELD, Eugene.HowISISelectsJornalsforCoverage. en: Scien ce and Technology Information Center. National Science Council. Taipei, Taiwan, 21-22, March, 1990. Philadelphia, PA, ISI, 1990. 19 p.

10. ARELlANO, Carlos; Carmen Pérez, PatricioBe rrios. Propuesta de un métododeevaluacióneditorialderevistascientíficasysuaplicación en revistas de medicina veterinaria de América Latina y el Caribe. Avances en Ciencias Veterinarias.Santiago, Chile, 6(2): 105-111, 1991.

11. ARBOLEDA, Orlando. Responsabilidad profesional en la producción de literatura bibliotecológica. Revista AIBDA, 2(1): 49-52, 1981. 


\section{BIBLIOGRAFIA}

ARBOLEDA, Orlando. Responsabilidadprofesionalenlaproducción deli teratura bibliotecológica. Revista AIBDA, 2(1): 49-52, 1981.

ARELLANO, Carlos; Carmen Pérez, Patricio Berrios. Propuesta de un métododeevaluacióneditorialderevistas científicasysuaplicación en revistas de medicina veterinaria de América Latina y el Caribe. Avances en Ciencias Veterinarias.Santiago, Chile, 6(2): 105-111, 1991.

BUDD, John.Publicaren biblio te cologíay cien cia de la in for ma ción.RevistaInteramericanadeBibliotecología, 12(2):71-91, Julio-Diciembre, 1989.

ESCUELADEBIBLIOTECOLOGIA. Literatura BibliotecológicaChilena 1980-1992. Santiago, I.P.S., 1992. 132p.

GARFIELD, Eugene. How ISI Selects Jornals for Coverage. en: Science and Technology Information Center. National Science Council. Taipei, Taiwan,21-22, March, 1990. Philadelphia,PA, ISI, 1990.19p.

GOMEZ, Héctor. Guía para la edición de publicaciones periódicas. Santiago, Ediciones Universidad Católica de Chile, 1985. 41 p.

HERBSTAEDT, Elfriede. Publicaciones seriadas científicas: un manual para su edición. Santiago, Chile, CONICYT, 1988. 56p.

HUERTA, Luis, Julia Leal. "Las revistas latinoamericanasde Bibliotecología y Ciencias de la In for ma ción y su re fle jo en ba ses de da tos bibliográficos". Seminario de título. Profesor Guía: Héctor Gómez. Santiago, Escuela de Bibliotecología, IPS, 1990. 55 p.

MARCO, Guy "Bibliographic con trol of Libraryand In formationScience Literature". Libri, 33(1): 45-60, March, 1983.

REUNION DE TRABAJO: "Presente y futuro de INFOBILA". México, D.F., 5 al 9 de Octubre, 1992. Informe México, D.F., CUIB-UNAM/IFLA, 1992.7 p.

RODRIGUEZ, Adolfo. "Las publicaciones periódicas de bibliotecología en lospaíses en de sa rrollo". InvestigaciónBibliotecológica:archivonomía, bibliotecologíaeinformación, 1(2):3-14, Ene ro-Ju lio, 1987. 